\title{
Autorank: A Python package for automated ranking of classifiers
}

\section{Steffen Herbold ${ }^{1}$}

DOI: $10.21105 /$ joss. 02173

\author{
1 Institute for Computer Science, University of Goettingen, Germany
}

\section{Software}

- Review ¿

- Repository ¿

- Archive cta

Editor: Arfon Smith प

\section{Reviewers:}

- OJonathanReardon

- Qejhigson

Submitted: 17 February 2020

Published: 14 April 2020

\section{License}

Authors of papers retain copyright and release the work under a Creative Commons Attribution 4.0 International License (CC-BY).

\section{Summary}

Analyses to determine differences in the central tendency, e.g., mean or median values, are an important application of statistics. Often, such comparisons must be done with paired samples, i.e., populations that are not dependent on each other. This is, for example, required if the performance different machine learning algorithms should be compared on multiple data sets. The performance measures on each data set are then the paired samples, the difference in the central tendency can be used to rank the different algorithms. This problem is not new and how such tests could be done was already described in the well-known article by Demšar (2006).

Regardless, the correct use of Demšar's guidelines is hard for non-experts in statistics. The distribution of the populations must be analyzed with the Shapiro-Wilk test for normality and, depending on the normality with Levene's test or Bartlett's tests for homogeneity of the data. Based on the results and the number of populations, researchers must decide whether the paired t-test, Wilcoxon's rank sum test, repeated measures ANOVA with Tukey's HSD as posthoc test, or Friedman's tests and Nemenyi's post-hoc test is the suitable statistical framework. All this is already quite complex. Additionally, researchers must adjust the significance level due to the number of tests to achieve the desired family-wise significance and control the false-positive rate of the test results.

Moreover, there are important aspects that go beyond Demšar's guidelines regarding best practice for the reporting of statistical result. Good reporting of the results goes beyond simply stating the significance of findings. Additional aspects also matter, e.g., effect sizes, confidence intervals, and the decision whether it is appropriate to report the mean value and standard deviation, or whether the median value and the median absolute deviation are better suited.

The goal of Autorank is to simplify the statistical analysis for non-experts. Autorank takes care of all of the above with a single function call. This is the difference between Autorank other packages, like Scipy (Virtanen et al., 2020), who expect users to know which tests to use and how to interpret the results. The decision flow of Autorank is as follows. 


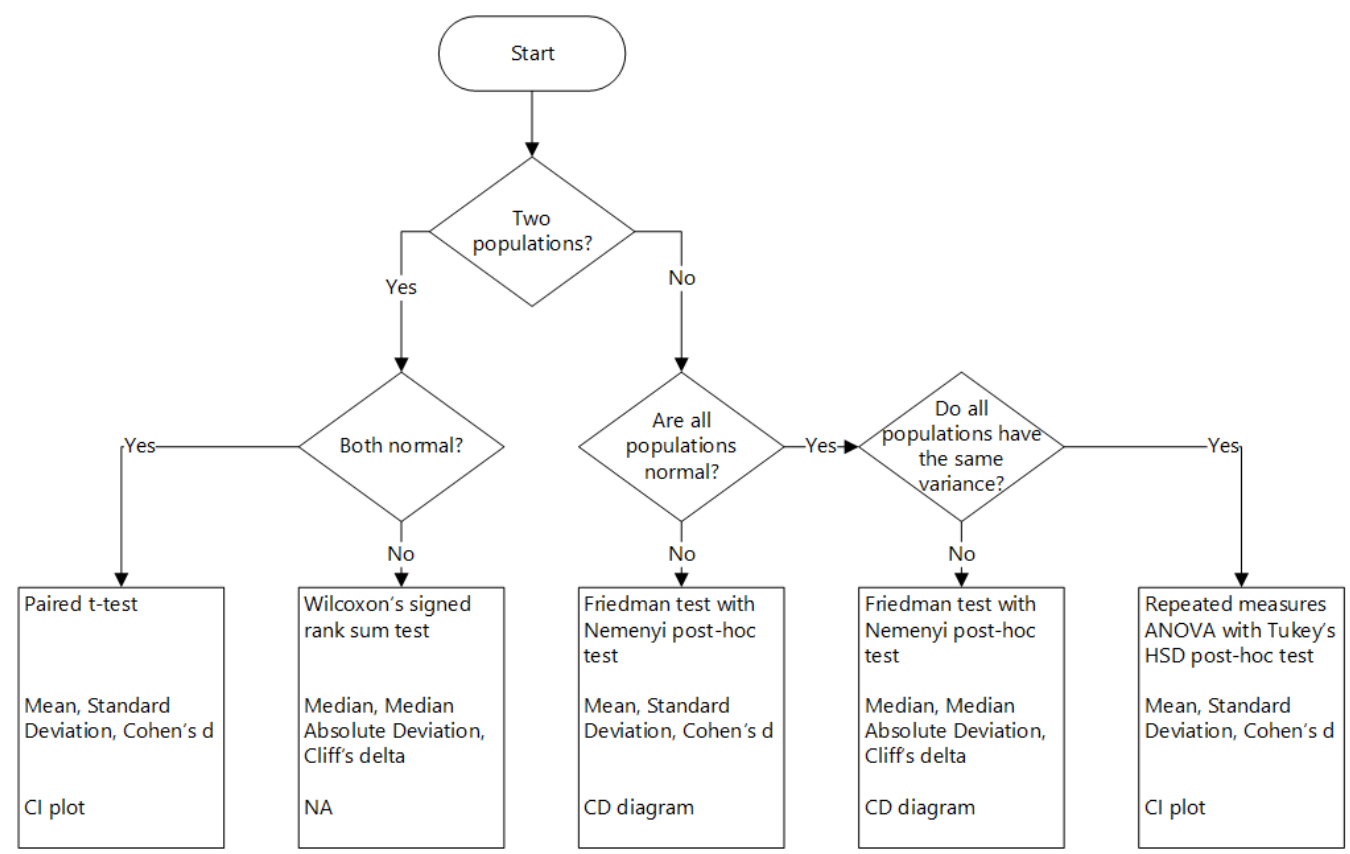

Figure 1: Decision Flow

Additional functions allow the generation of appropriate plots, result tables, and even of a complete latex document. All that is required is the data about the populations is in a dataframe.

We believe that Autorank can help to avoid common statistical errors such as, such as the use of inappropriate statistical tests, reporting of parametric measures instead of non-parametric measures in case of non-normal data, and incomplete reporting in general.

\section{Using Autorank}

In our research, we recently used autorank to compare differences between data generation methods for defect prediction research (Herbold, Trautsch, \& Trautsch, 2020). In general, Autorank can be used anywhere, where different classifiers are compared on multiple data sets. The results must only be prepared as a dataframe. For example, the dataframe could contain the accuracy of classifiers trained on different data sets. ${ }^{1}$ The following three lines would then perform the statistical tests, generate the textual description of the results, as well as the plot of the results.

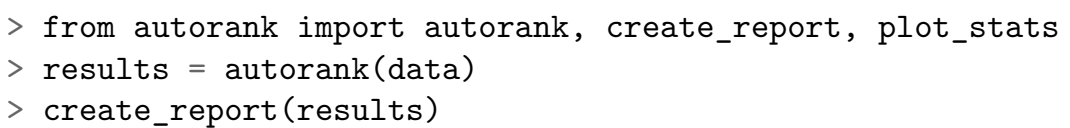

The statistical analysis was conducted for 6 populations with 20 paired samples.

The family-wise significance level of the tests is alpha $=0.050$.

We rejected the null hypothesis that the population is normal for the population Random Forest $(p=0.000)$. Therefore, we assume that not all populations are normal.

Because we have more than two populations and the populations and one of

\footnotetext{
${ }^{1}$ See also: https://github.com/sherbold/autorank/tree/master/examples/sklearn.py
} 
them is not normal, we use the non-parametric Friedman test as omnibus test to determine if there are any significant differences between the median values of the populations. We use the post-hoc Nemenyi test to infer which differences are significant. We report the median (MD), the median absolute deviation (MAD) and the mean rank (MR) among all populations over the samples. Differences between populations are significant, if the difference of the mean rank is greater than the critical distance $\mathrm{CD}=1.686$ of the Nemenyi test.

We reject the null hypothesis $(p=0.000)$ of the Friedman test that there is no difference in the central tendency of the populations Naive Bayes $(\mathrm{MD}=0.875+-0.065, \mathrm{MAD}=0.053, \mathrm{MR}=2.750)$, Random Forest $(\mathrm{MD}=0.850+-0.100$, $M A D=0.062, M R=2.850), R B F$ SVM $(M D=0.885+-0.217, M A D=0.059, M R=2.900)$, Neural Net $(M D=0.876+-0.070, M A D=0.045, M R=3.300)$, Decision Tree $(\mathrm{MD}=0.810+-0.173, \mathrm{MAD}=0.074, \mathrm{MR}=4.525)$, and Linear SVM (MD=0.710+-0.245, $M A D=0.253, M R=4.675)$. Therefore, we assume that there is a statistically significant difference between the median values of the populations. Based the post-hoc Nemenyi test, we assume that there are no significant differences within the following groups: Naive Bayes, Random Forest, RBF SVM, and Neural Net; Random Forest, RBF SVM, Neural Net, and Decision Tree; Neural Net, Decision Tree, and Linear SVM. All other differences are significant.

$>$ plot_stats (data)

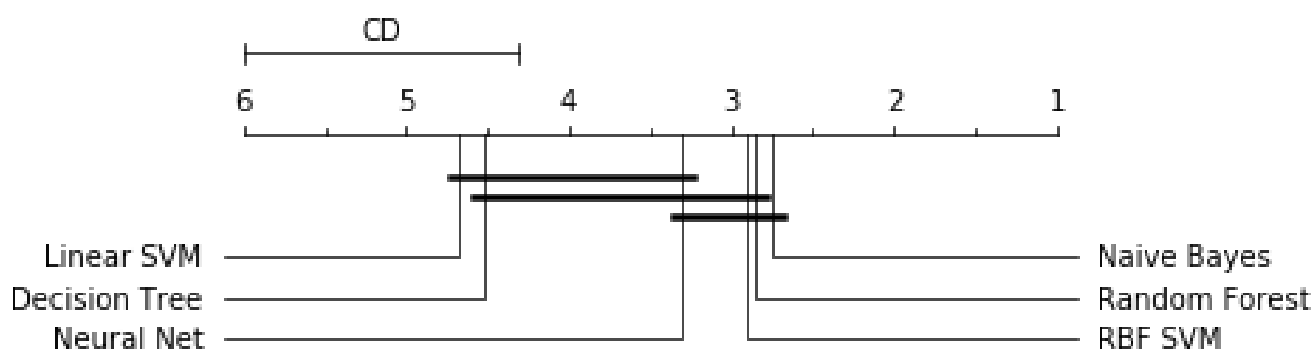

Figure 2: CD Diagram

\section{Acknowledgements}

This work is partially funded by DFG Grant 402774445.

\section{References}

Demšar, J. (2006). Statistical comparisons of classifiers over multiple data sets. J. Mach. Learn. Res., 7, 1-30.

Herbold, S., Trautsch, A., \& Trautsch, F. (2020). Issues with szz: An empirical assessment of the state of practice of defect prediction data collection. Retrieved from http://arxiv. org/abs/1911.08938 
JesS

Virtanen, P., Gommers, R., Oliphant, T. E., Haberland, M., Reddy, T., Cournapeau, D., Burovski, E., et al. (2020). SciPy 1.0: Fundamental Algorithms for Scientific Computing in Python. Nature Methods, 17, 261-272. doi:10.1038/s41592-019-0686-2 